\title{
La recrianza humanizada: Un giro a las relaciones de poder y al paradigma adultocéntrico en las instituciones de protección de niños, niñas y adolescentes en situación de vulneración de derechos
}

\author{
Humanized Upbringing: A Turn to Power Relations and the Adult-Centered Paradigm in \\ Institutions for the Protection of Children and Adolescents in Situation of Violation of Rights
}

\begin{abstract}
Reeducação infantil humanizada: Uma volta nas relações de poder e no paradigma adultocêntrico nas instituições de proteção de crianças e adolescentes em situação de vulneração de direitos
\end{abstract}

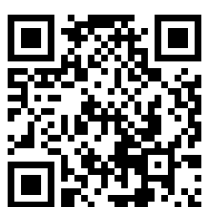

\author{
Armando Zuluaga-Gómez \\ Alcaldía de Medellín, Unidad de Niñez \\ Medellín-Colombia \\ armandozgom@gmail.com \\ http://orcid.org/0000-0001-8286-5152
}

Recibido • Received • Recebido: 25 / 08 / 2016

Corregido • Revised • Revisado: 07 / 12 / 2017

Aceptado • Accepted • Aprovado: 29/ $01 / 2018$

\begin{abstract}
Resumen: Esta reflexión tiene como base las notas consignadas en un diario de campo y su objetivo es sistematizar la experiencia adquirida como educador en el Centro de Diagnóstico y Derivación, operado por la Universidad de Antioquia a través del Proyecto Crecercon Dignidad (Zuluaga, 2015-2016), adscrito a la Unidad de Niñez, en la ciudad de Medellín-Colombia, cuyo propósito es la protección inmediata de niños, niñas y adolescentes en situación de vulneración de derechos. Analizaremos aquí las relaciones de poder que se establecen al interior del paradigma adultocéntrico, develaremos en estas la génesis del maltrato a la niñez y veremos cómo dichas prácticas normalizadas en la crianza de la niñez, por parte de sus familias de origen, permean las instituciones de protección que han sido creadas para llevar a cabo los procesos de restablecimiento de derechos. Cuando se instituyen y legitiman relaciones de poder desiguales al interior de la familia, se consolida la hegemonía de las personas adultas sobre la niñez, que terminan siendo objetivados, normalizándose de este modo su maltrato. Dichos paradigmas relacionales son también susceptibles de reproducirse en las instituciones educativas, incluidas aquellas destinadas a la protección de la niñez en situación de vulneración de derechos. Plantearemos una propuesta denominada recrianza humanizada, la cual es sugerida para la conducción grupal en las instituciones de protección, tarea encomendada a agentes educativos.
\end{abstract}

Palabras claves: Paradigma adultocentrico; relaciones de poder; maltrato a la niñez; re crianza humanizada; agente educativo; institución de protección; restablecimiento de derechos.

\footnotetext{
${ }^{1}$ Estudiante de psicología. Diplomas: De la atención a la interacción con niños, niñas y adolescentes en situación de vulnerabilidad. Universidad de Antioquia. 2012. De lo invisible a lo visible en la explotación sexual comercial de niños, niñas y adolescentes. Facultad Nacional de Salud Pública, Universidad de Antioquía.2015.
} 
doi: http://dx.doi.org/10.15359/ree.22-2.20

URL: http://www.una.ac.cr/educare

CORREO: educare@una.cr

\begin{abstract}
This reflection is based on the notes recorded in a field journal and its objective is to systematize the experience acquired as an educator in the Diagnostic and Derivation Center, operated by the University of Antioquia through the Grow with Dignity Project (Zuluaga, 2015-2016), attached to the Unit of Childhood, in the City of Medellín, Colombia, whose purpose is the immediate protection of children and adolescents in situations of violation of rights. We will analyze, here, the power relations that are established within the adult-centered paradigm; we will reveal the genesis of child abuse in these relations, and we will see how these normalized practices in the upbringing of children by their families of origin permeate the protection institutions that have been created to accomplish processes of restoration of rights. When unequal power relationships are instituted and legitimated within the family, the hegemony of adults over childhood is consolidated, and the latter ends up being objectified, like this normalizing their abuse. These relational paradigms are also susceptible to reproduction in educational institutions, including those aimed at the protection of children in situations of violation of rights. We will suggest a proposal called humanized reeducation, which is indicated for group leadership in protection institutions, a task entrusted to educators.
\end{abstract}

Keywords: Adult-centered paradigm; power relationships; abuse of children; humanized reeducation; educational agent; protection institution; restoration of rights.

Resumo: Esta reflexão é baseada em notas registradas em um diário de campo e tem como objetivo sistematizar a experiência adquirida de um educador no Centro de Diagnóstico e Referência, operado pela Universidade de Antioquia, através do Projeto Crescer com Dignidade (Zuluaga, 20152016), ligado a Unidade de Infância em Medellín , Colômbia, cuja finalidade é a proteção imediata de crianças e adolescentes em situações de violações de direitos. Analisaremos aqui as relações de poder que se estabelecem dentro do paradigma adultocêntrico, descobriremos nestas a gênese de maltrato para com as crianças e veremos como estas práticas são vistas como normais na educação das crianças, por parte de suas famílias de origem, e permeiam também as instituições de proteção que foram criadas para levar a cabo os processos de assegurar os direitos. Quando as relações de poder desiguais dentro da família são criadas e legitimadas, a hegemonia dos adultos sobre as crianças é reforçada, e estas acabam sendo objetivadas, normalizando assim a prática da violência. Tais paradigmas relacionais também são susceptíveis de se reproduzir em instituições educacionais, incluindo as que são destinadas à proteção das crianças em situações de violações de direitos. Apresentaremos uma proposta chamada Reeducação infantil humanizada, que será sugerida para a direção grupal nas instituições de proteção, uma tarefa confiada aos educadores.

Palavras-chave: paradigma adultocêntrico; relações de poder; crueldade para com as crianças; reeducação humanizada; agente educativo; instituição de proteção; restauração dos direitos. 
Los niños y niñas no son mini-seres humanos con mini-derechos humanos. Pero mientras los adultos continúen considerándolos como tales, la violencia contra los niños y niñas persistirá.

-(Maud de Boer-Buquicchio, Secretaria General Adjunta del Consejo de Europa, Estrasburgo, 2006 en Pinheiro, 2006, p. 4)

\section{Introducción}

En Colombia el Estado ha confiado a instituciones de carácter público o privado la responsabilidad de atender las disposiciones de las defensorías de familia en los procesos administrativos de restablecimiento de derechos de niños, niñas y adolescentes (NNA) que han sido víctimas de algún tipo de maltrato, violencia física, psicológica, sexual u otro tipo de vulneración de sus derechos. En algunas de esas instituciones, de manera no consciente, subsisten actitudes, formas de relacionarse y prácticas propias del paradigma adultocéntrico/patriarcal.

En este texto adoptaremos la definición de la Unicef (2006) en la Convención sobre los derechos del niño, articulo 1, el cual entiende por niño "todo ser humano menor de dieciocho años de edad, salvo que, en virtud de la ley que le sea aplicable, haya alcanzado antes la mayoría de edad" (p. 10) y utilizaremos indistintamente la sigla NNA (niños, niñas y adolescentes) para referirnos a este grupo.

El modelo adultocéntrico es un tipo de crianza basado en concepciones de las personas adultas como proyectos terminados, dueñas de la experiencia y la verdad, la que han conseguido a través de sus años de vida; lo cual supone, para estos individuos, una posición superior y hegemónica respecto de la niñez.

Lo dicho anteriormente se refleja en las relaciones establecidas entre estos dos grupos poblacionales y en la forma en la cual las personas adultas cumplen su función de transmisión de la cultura, valores y estilos de vida, función denominada en términos generales como crianza.

Al considerar la supuesta superioridad de los grupos de "mayores", el adultocentrismo anula las necesidades, sentimientos y opiniones de los "chicos o chicas". Arévalo (1996) define este como:

Una categoría que designa en nuestras sociedades una relación asimétrica y tensional de poder entre los adultos... y los..., [que no lo son], visión de mundo... montada sobre un universo simbólico y un orden de valores propios de la concepción patriarcal. (pp. 62, 58)

Lo anterior nos introduce en el problema central de la presente reflexión: las relaciones de poder entre personas adultas y NNA, en las cuales se fraguan el abuso y el maltrato hacia la niñez, según nuestro punto de vista. 
Cuando son instituidas estructuralmente, legitimadas y reproducidas relaciones de poder asimétricas entre las personas adultas y la niñez, al interior de instituciones como la familia, la escuela, las organizaciones, etc. se consolida la hegemonía de los sujetos adultos sobre los NNA, terminando por objetivar estos últimos y muchas veces justificando las violencias hacia niños, niñas y adolescentes en la buena intención de formarles como "personas de bien", invisibilizándoles como sujetos.

Desde estos parámetros, los grupos NNA quedan expuestos al maltrato; pues supuesta su inferioridad, los niños y las niñas deben ser objeto de dirección y control, sin un reconocimiento como sujetos en otredad, dando por entendido que "no son actores de su propio desarrollo y son solo proyectos de personas adultas" (Posada-Díaz, Gómez-Ramírez y Ramírez-Gómez, 2014, p. 10).

\section{Marco teórico}

Es importante implicar de manera directa a los NNA que han sido víctimas de cualquier forma de maltrato, en la gestión para el restablecimiento de sus derechos, humanizando este proceso y dándoles, en el mismo, un lugar protagónico, evitando así considerarles como meros entes receptores de unas acciones, que verán como ajenas y exógenas, lo cual incrementa su resistencia y evasión de las medidas tomadas para restablecer sus derechos, situación recurrente en este tipo de procesos.

Se debe reconocer y resignificar a NNA como una víctima genuina del abuso de poder de las personas adultas-victimarias y empoderarles como tales, merecedoras también de los derechos que le han sido reconocidos a otras víctimas de violencias.

Los grupos NNA en situación de maltrato deben acceder al derecho a la justicia y la reparación; es en esto en lo que el Estado de Colombia se encuentra en mayor mora, ya que casi nunca los sujetos victimarios son castigados por sus delitos, como parte integral de la reparación a sus víctimas.

En este punto se evidencia como los NNA son, en nuestra sociedad colombiana, "niño sacer", en el sentido en que Bustelo (2007, p. 26) parafrasea el concepto de Agamber "homo sacer", para referirse a ese ser humano a quien en la práctica se le despoja de valor político y ciudadano, al quedar impunes las violencias ejercidas sobre él; tal y como crudamente protestaba una adolescente quien se encontraba en una institución de protección: "ese tío mío me violó y la que resulto pagando el encierro fui yo".

Castigar ejemplarmente a los grupos victimarios es responsabilidad de un Estado social de derecho, en el que se tiene como valor con carácter real y no una demagogia, la prevalencia de los derechos y el interés superior sobre los derechos de la niñez. 
El proceso de reconocimiento como víctimas exige legitimarles en su otredad, su realidad individual, su ciclo vital; evitando buscar en esto alguna razón para justificar las violencias hacia los niños y niñas, como pretenden los sujetos agresores: "es que ese muchacho es muy rebelde y no entiende por las buenas", lo que se escucha frecuentemente cuando se constatan los casos de maltrato.

La cicatrización de las heridas no solo físicas, sino emocionales, toma su tiempo; este es un proceso gradual cuyos plazos deben desarrollarse de acuerdo con las necesidades de los NNA y no con parámetros e indicadores externos; debe llevarse a cabo una reflexión crítica sobre lo sucedido, posibilitada con la intermediación de dispositivos pedagógicos como el arte, el juego y la arteterapia, entre otras.

Teniendo como referencia lo que sucede con respecto a los delitos sexuales, pese al rechazo y el repudio social que estos generan, los niveles de impunidad son dramáticos; consideramos que no se han reconocido a NNA en su condición de víctimas y su derecho a la justicia no ha sido garantizado, quedando así en franca inobservancia sus derechos por parte del Estado colombiano y, en consecuencia, se han dejado de restablecer efectiva e integralmente.

De acuerdo con los pediatras sociales Posada-Díaz et al. (2014):

En estudios específicos sobre el neurodesarrollo y la salud mental se ha encontrado que los niños y niñas acompañados con maltrato en la primera infancia se caracterizan porque tienen con mayor frecuencia trastornos del apego y la socialización, estrés postraumático y traumatismos emocionales intensos y alteración de la capacidad de tejer resiliencia, lo cual se manifiesta en la pre adolescencia, la adolescencia y la adultez como comportamientos violentos, delincuencia, abuso sexual, adicciones y violencia de pareja. (p. 25)

Recuperarse de las secuelas físicas dejadas por el maltrato y la negligencia en el cuidado requiere tiempo suficiente y cuidados terapéuticos pertinentes, lo cual exige ir al ritmo propio de NNA que se encuentran en proceso de restablecimiento de derechos.

No se trata de criticar per se las normas y pactos de convivencia instaurados para que funcionen las instituciones de protección para NNA, ni de quedarse en la lastimera demanda de quienes piensan que " hay que dejarlos hacer lo que quieran por su situación" sino de comprenderles en su realidad y lograr su resocialización acompañándoles con amor en la introyección de la norma, como una postura ética que facilita el buen vivir y no por temor a las consecuencias y la reacción de la persona adulta.

Barudy (1998), en su texto El dolor invisible de la infancia, refiere cómo el maltrato genera en NNA manifestaciones traumáticas en diferentes áreas: en lo afectivo, se compromete la emocionalidad, la autoestima y la empatía; a nivel cognoscitivo, se dan disociaciones cognitivas y de la identidad; en lo conductual, se presentan comportamientos autodestructivos como el 
doi: http://dx.doi.org/10.15359/ree.22-2.20

URL: http://www.una.ac.cr/educare

CORREO: educare@una.cr

consumo de SPA, violencia, sexualidad insegura, delincuencia, etc.; en lo relacional se encuentran dificultades en cuanto al apego y la vinculación, así mismo la capacidad de reflexión y decisión ética y moral resulta afectada.

En el presente texto entenderemos por maltrato todo acto relacional entre niñez y personas adultas, donde se demuestre cualquier forma de abuso sutil o evidentemente violento, en el que la persona adulta, considerándose en una posición de superioridad, constriñe arbitrariamente, de manera física, psicológica o sexual, a un NNA o se muestre negligente frente a la garantía de sus derechos, afectando con esto su desarrollo físico o psicosocial.

Son manifestaciones de maltrato hacia la niñez: las reprimendas violentas; todas las formas de violencia o abuso sexual; las humillaciones psicológicas; la negligencia en su cuidado; el desinterés en la protección y garantía de sus derechos; la explotación sexual; su utilización o instrumentalización dentro del conflicto armado o la delincuencia, el grito, la intimidación o amenazas; la sobreexigencia (esperar que un niño o una niña de quince meses logre el control total de sus esfínteres); la deprivación afectiva y la precariedad económica que no facilite el desarrollo integral de NNA en un ambiente sano y favorable.

Una forma de maltrato sutil por sobreexigencia bastante común en las instituciones de protección se da cuando se le ordena, a una persona NNA, que se vincule y participe desde el principio de todas las actividades del diario vivir $^{2}$ sin considerar su situación, que puede incluir un estrés postraumático, desconfianza hacia las personas adultas, depresión, irritabilidad e incluso un síndrome de abstinencia por consumo de sustancias psicoactivas, síntomas que necesitarán de un periodo inicial de reposo y que no suele darse a los grupos NNA en las instituciones.

El juego libre y las expresiones artísticas han sido considerados un buen método para aprender cualquier concepto o valor con NNA, ya que posibilita la construcción de reflexiones conjuntas, la introyeccion de la norma y de posturas éticas; todo ello necesario en la realización de proyectos de vida, en el marco del desarrollo humano y la convivencia social.

Por lo anterior, en las instituciones de protección algunas cosas se enseñan jugando, (deberían ser todas); sin embargo, hace falta que la participación de NNA se dé espontáneamente y los espacios para jugar no sean percibidos por ellos o ellas como una imposición o un intento más de mantenerles bajo control. Un verdadero disfrute es, sin lugar a dudas, el prerrequisito para sacar del juego un buen provecho.

El juego como derecho debe estar siempre garantizado, como debió estarlo el afecto y la protección desde los primeros momentos de la vida; la posibilidad de jugar en el ámbito institucional, donde se llevan a cabo los procesos de restablecimiento de derechos, debe

\footnotetext{
${ }^{2}$ Cronograma de actividades para el día en una institución.
}

6 
mantenerse ofertada, no como necesidad del agente educativo de ocuparles para controlarles y contenerles, sino siempre ofrecido como un seno grávido de leche, a la espera activa y paciente del deseo del niño o la niña.

Jugar no puede ser una exigencia ni objeto de evaluación, ya que de este modo se corre el riesgo que este sea un mecanismo útil al discurso hegemónico, en el modelo de crianza adultista, para tejer habilidosamente las desigualdades entre personas adultas y niñez.

El juego libre y las expresiones artísticas como dispositivos pedagógicos permiten repensar y resignificar las relaciones de poder que necesariamente se gestan entre los individuos NNA y las personas adultas, ya que este elemento (el poder), emerge inevitablemente al interior de las dinámicas relacionales de los seres humanos.

Para Foucault (1977) el poder no es un atributo o una cualidad de las personas o grupos, sino una característica dada per se en las relaciones sociales. Por lo tanto, la salida difiere de negar que este se da en las dinámicas relacionales entre personas adultas y NNA, ni deponer las figuras de autoridad, sino la búsqueda del equilibrio, la solidaridad, la tolerancia y la democracia en las pautas de crianza, donde cada quien cumpla con su rol y las funciones de este mismo, con la guía no por el poder como razón, sino en la razón del poder, de acuerdo con la inquietud de los movimientos sociales latinoamericanos en la década de los setenta, recogida por Baró.

Es posible enseñar a NNA por medio del juego a no lamentarse, y enfrentar la vida como se ha presentado hasta ahora, aceptando el reto de deconstruirla para emprender la tarea de resignificarse y construir una nueva existencia posible, enseñar al niño y a la niña a valerse del tejido institucional y personal para catapultarse como un ser fuerte y resiliente, que reconquista y exige sus derechos, y estimula su autonomía.

¿Qué hacercon la niñez en situación de maltrato que se niega a jugar? En un primer momento es preciso concederle lo que se le ha negado, es decir: tolerancia, paciencia y comprensión. Es necesario, al interior del ámbito institucional, atraerle y esperarle, a la manera en que De SaintExupéry (2009) lo describe en el principito, personaje que primero debe domesticar al zorro, con el fin de hacerse familiar y amigable y, posteriormente, poder jugar con él.

\section{Discusión de ideas}

Es preciso entender que estos grupos de NNA no tuvieron las condiciones ni el tiempo necesario para su pleno desarrollo afectivo y social; es preciso que quienes hemos asumido la tarea de acompañar la resignificación de su crianza, esperemos que germine en estos el sentimiento de auto y hetero confianza; puesto que confiar en las demás personas es una construcción progresiva, edificada con el acompañamiento de individuos adultos protectores y afectivos; y no se da como resultado de generación espontánea al cruzar el umbral de la puerta de una institución de protección. 
Más que una meta, un derecho es un viaje personal, emprendido desde dos frentes: el íntimo y el social; por lo tanto, un derecho es una conquista de la sociedad y de cada sujeto; puede ser considerado una utopía en que la exigencia o el reclamo social e individual es lo que llamaríamos empoderamiento, un elemento fundamental para el pleno restablecimiento de los derechos de NNA en situación de maltrato.

El empoderamiento sobre los derechos debe ser provisto de la capacidad de tolerar la frustración, ya que no podemos desconocer los condicionantes sociales que afectan su ejercicio; sin embargo, como afirma Hessel (2010): "No hay que transigir con los derechos" (p. 7).

Bauman (2004), en su obra Modernidad líquida, la cual podríamos denominar como un análisis filosófico y sociológico de la época actual, o como él la llama, era líquida, hace exhaustivos hallazgos en la sociedad contemporánea sobre esos condicionantes sociales, sin que el autor los presente con ese concepto especifico.

La respuesta a la pregunta: ¿Los niños y niñas nacen como sujetos o son producto de la crianza?, nos dará pistas de la concepción que tenemos de NNA y de la etimología de las problemáticas que les aquejan.

El autor de este texto cree firmemente que el desarrollo humano y el proyecto de vida de la totalidad de NNA son resultado de la crianza, la educación, la socialización, la relación con las personas adultas significativas, las influencias o afectaciones del contexto y la época; también en este proceso intervienen las características de un temperamento innato.

Este asunto es complejo y no deja de resonar en mí la pregunta por la responsabilidad de los propios sujetos NNA en su formación; no puedo evitar el marasmo mental y el dilema ético que este interrogante me suscita, sobre todo cuando estamos hablando de NNA cuya historia ha sido marcada con el maltrato, a quienes esta reflexión pretende reconocerles en el lugar de víctimas, evitando con esto que les siga catalogando como personas subversivas y causantes de su propia situación, por parte del modelo adultocéntrico.

La condición de víctimas debe otorgar a NNA el derecho a la reparación, que tiene como condición un acompañamiento afectuoso, mediado por nuevas relaciones con las personas adultas, basado en la solidaridad y no en el poder.

Un proceso de restablecimiento de derechos que rehabilite afectiva y efectivamente a NNA en el ejercicio pleno de estos debe restituir en ellos la dignidad, la salud, la autoestima, la autonomía, la creatividad, la felicidad y la solidaridad. Debe renovar su capacidad de resiliencia frente a un contexto frecuentemente aversivo a sus proyectos de vida; un proceso que podríamos denominar, parafraseando al grupo de puericultura de la Universidad de Antioquia, como recrianza humanizada. 
En la recrianza humanizada debemos escuchar atenta y conscientemente a NNA, conectándonos con sus historias de carencias, negaciones y heridas, las que permanecen como cicatrices o costras en su ser y piel. Debemos además tomarnos en serio lo que piensan con la intención de volver en acciones sus palabras, en beneficio propio y de los otros sujetos; haciendo efectivo su derecho a la participación.

En este proceso, sus acompañantes del ámbito educativo y psicosocial deben (debemos) estar conscientes de la responsabilidad de volver y actuar sobre heridas abiertas o mal cicatrizadas, que aún les duelen, lo que exige una alta dosis de consentimiento, afecto, consideración, tolerancia y comprensión.

\section{Liderazgo prosocial para agentes educativos y facilitadores de grupos de recrianza humanizada en instituciones de protección}

Para que la participación de NNA en sus procesos produzca un verdadero aprendizaje significativo, adecuado a la recrianza humanizada, el agente educativo deberá mantener un liderazgo prosocial, el cual fomente la autonomía, la responsabilidad y la introyección de la norma.

La conducción de un grupo conformado por NNA con derechos vulnerados por cualquiera de las formas de maltrato, abuso, violencias o negligencia en una institución de protección implica orientarles en la conquista del mayor grado de autonomía posible, su afirmación positiva como personas que asumen con responsabilidad sus proyectos de vida y la capacidad de mantener adecuados niveles de convivencia y participación en las decisiones o acciones que les afectan.

El primer paso es la apertura, por parte de la Defensoría de Familia de un proceso administrativo de restablecimiento de derechos, que en algunas oportunidades requiere la ubicación de NNA en una institución transitoria o especializada, donde, en medio de la dinámica grupal, se gesta su resocialización en una perspectiva de desarrollo humano.

Los procesos grupales en las instituciones de protección involucran, en su dinámica: relaciones de poder, dependencia respecto al agente educativo, demanda de graduales niveles de autonomía, momentos de crisis, afectaciones por las características y crisis personales de sus miembros, las cuales alteran la convivencia grupal; así mismo dificultades en la comunicación, resistencias al cambio y en muchos casos rigidez excesiva de la estructura normativa institucional.

El agente educativo debe ser conocedor de los elementos mencionados y tener la pericia para interactuar con estos, canalizándolos hacia la consecución de los objetivos.

En adelante analizaremos cada una de estas variables con la intención de promover la reflexión y cualificación de los agentes educativos, pero también de los demás actores que interactúan con NNA, a quienes se ha decretado una medida de ubicación institucional en un centro de protección. 


\section{Relaciones de poder}

Al interior de este tipo de grupos se dan, al menos, dos roles instituidos: el agente educativo y el grupo NNA beneficiario del programa; estos presuponen una posición de poder respecto del otro, con unas funciones preestablecidas y un margen de acción igualmente predeterminado.

Las funciones del agente educativo son: conducir el grupo, representar la autoridad, instaurar la norma y sancionar su incumplimiento, administrar los recursos puestos a disposición de las personas beneficiarias, tanto tangibles (implementos de distinta índole) como intangibles (tiempos, actividades, etc.). Y contener los esfuerzos de I NNA por desafiar o fijar posiciones instituyentes frente a las normas, figuras de autoridad y el sistema institucional.

Al agente educativo se le ha dado autoridad sobre NNA, lo cual es necesario para cumplir con sus funciones y ayudar a mantener lo instituido en el modelo o plan de atención integral (PAI) Este debe ser diestro en su manejo, equilibrando la relación de poder y evitando el autoritarismo, el constreñimiento o la coerción a la autonomía y la participación de NNA. Más adelante volveremos en el análisis de este aspecto.

Es importante no caer en la reproducción del adultocentrismo y lograr un movimiento pendular entre la intervención (relación vertical) y la interacción (relación horizontal), dicho movimiento es producto de la lectura permanente que debe realizar el agente educativo de las dinámicas individuales y grupales que están bajo su conducción.

Esta variable (la relación de poder) es transversal a todos los elementos que están presentes en los procesos grupales y afecta sistémicamente las dinámicas que se dan en su interior.

La autoridad no debe ser un dispositivo que alimente el narcicismo del agente educativo o mantenga la inequidad entre personas adultas y NNA; por el contrario, está servirá como estrategia para acompañarles en sus procesos de recrianza e introyección de la norma, el restablecimiento de sus derechos y su afirmación como sujetos.

\section{Dependencia respecto al personal educador}

Los grupos tienden a generar dependencia del agente educativo, ya que este orienta las acciones necesarias para la consecución de las metas, en esta figura se busca descargar la responsabilidad en la toma de decisiones, entre otras cosas porque se le admira, se le supone un saber, se le reconoce como líder o se subordina a su autoridad.

Los grupos de NNA modulan ciertas conductas o se sienten en libertad de manifestar otras, cuando el agente educativo está inmediatamente presente. En relación con lo anterior, en ciertos momentos de la vida grupal se dan cambios, más que por convicción, por conformidad. 
Según Kelman (citado por Ovejero, 2007): Existen tres tipos de conformidad, entre estas la simulada:

Consiste en aceptar de forma pública un comportamiento o un sistema de valores sin adherirse a ellos de forma privada. En apariencia los individuos o los grupos se someten a fin de evitar ciertos agravios: devaluación, represión, etc. Pero conservan sus creencias y están dispuestos a cambiar su comportamiento desde el momento en que las circunstancias no se lo impongan". (p. 164)

Se modifican actitudes y comportamientos, sin introyectar realmente la norma, esto último imperioso en el proceso de socialización.

Es necesaria la presencia del agente educativo como ente observador participante en los diferentes espacios, quien mantiene una lectura constante sobre la convivencia y, con base en esto, toma la decisión de cohesionar u otorgar autonomía al grupo.

La demanda de orientación de NNA es alta; todo el tiempo están solicitando directrices, sobre los modos de proceder en los diferentes espacios y momentos; asunto que debe aprovecharse para dirigir preguntas reflexivas, que estimulen su autonomía: ¿Qué crees que debe hacerse en este momento o caso?

La autonomía es posible cuando el agente educativo permite a NNA tomar decisiones, asumir las consecuencias de sus conductas y suspende temporalmente la emisión de directrices, permitiendo que actúen con libertad, apostando por sus elecciones; descartando por momentos el control exógeno e incentivando el autocontrol. De igual modo, en los espacios de tiempo libre conviene disponer de una metodología llamada centros de interés, la que permite una división espontánea en subgrupos de acuerdo con los gustos, predilecciones e identificación de NNA con sus pares, con quienes comparte sus intereses.

\section{Momentos de crisis}

En algún momento los grupos de NNA pueden asumir conductas disruptivas, el agente educativo deberá entonces recoger el grupo y cohesionarlo nuevamente, recuperando el control sobre este mismo, para lo que puede necesitar del apoyo de otros actores institucionales externos al grupo, como líderes pedagógicos y equipos psicosociales. Deberá mantenerse el control, hasta que se den las condiciones para un nuevo ejercicio de autonomía. La metáfora de las cometas nos resulta útil para explicar lo anterior, ya que para lograr que una cometa se eleve por los aires, es necesario un equilibrio entre el "tire y afloje" de la pita, pues cuando quien intenta elevarla solo "tira" de esta sin soltar, corre el riesgo que se reviente; o cuando solo afloja y suelta, esta puede ser atrapada por las corrientes de aire; en cualquiera de los dos casos la cometa se pierde. 
doi: http://dx.doi.org/10.15359/ree.22-2.20

URL: http://www.una.ac.cr/educare

CORREO: educare@una.cr

\section{Resistencia al cambio}

Generalmente las instituciones valoran y sostienen los grupos sobre el control; el imaginario es que si no hay alteración en este las cosas van bien; sin embargo, la represión, como ha quedado explicado, va en menoscabo de la autonomía de NNA y desconoce la resistencia al cambio, natural dentro de los grupos y los procesos de transformación personal.

Una dosis de caos, crisis recurrentes y la negativa a seguir las pautas institucionales deben ser reconocidas y manejadas con destreza, volviéndolas una oportunidad e insumo para motivar los procesos de cambio.

\section{La comunicación}

La comunicación humana es el dispositivo que regula la convivencia, motiva el logro de las metas, transfiere valores y actitudes, y ayuda a mantener la cohesión en los grupos. Además de algunas metodologías artísticas, simbólicas, deportivas y lúdicas, en las que se hace uso del lenguaje no verbal y que tienen todas ellas una función educativa, la palabra es un hilo conductor privilegiado en las instituciones, para orientar y acompañar los procesos de NNA; por lo cual su uso debe ser cuidadoso, claro y propositivo por parte del agente educativo, quien tendrá constantemente que mejorar los canales y contenidos de comunicación, a fin de mantener adecuados niveles de efectividad de esta, afrontar como retos las dificultades usuales que se presentan entre NNA y entre este grupo y el agente educativo.

\section{Rigidez en la estructura institucional}

En instituciones que atienden adolescentes, y de acuerdo con como estos se comportan moralmente, el sistema institucional obedece a los siguientes aspectos:

Deben existir normas básicas no negociables, que hacen referencia a valores o principios cardinales de convivencia, respeto de cada persona por sí misma y por las otras, así como por valores sociales aceptados. Teniendo en cuenta las historias de NNA, para quienes presentan dificultades en la socialización, las normas deben ser reaprendidas e introyectadas en el marco de un proceso.

Las normas institucionales deben tener un sentido explicable para adolescentes, quienes deben comprender cómo estas los benefician y facilitan su convivencia con los demás individuos.

Excepto las normas básicas, algunas otras pueden ser negociables, lo cual garantiza la participación de NNA en los asuntos que les afectan, se aporta, de este modo, a su madurez emocional y social. 
Algunas normas que son útiles en ciertos momentos y espacios pueden ser sustituidas en otros para facilitar las cosas, previo acuerdo o conciliación con el grupo, lo que genera autonomía.

\section{Conclusiones}

La génesis estructural del maltrato a la niñez se encuentra en la disparidad subyacente en las relaciones de poder, establecidas entre personas adultas, niños y niñas, al interior del modelo adultocéntrico; esto, con la filigrana que Foucault (1979) llamaría la microfísica del poder.

Manifestaciones comportamentales como la violencia, rasgos o características de personalidad como oposicionista desafiante, la anomia, la delincuencia, las adicciones, las prácticas de sexualidad insegura, no son propiamente decisiones vitales tomadas en libertad por los grupos NNA, sino que son más bien las consecuencias de un proceso de crianza marcado por la negligencia, el maltrato y el abuso.

La resignificación de la relación de poder natural entre el agente educativo y el niño, niña o adolescente solo puede lograrse al renunciar a una excesiva rigidez institucional e inclusive personal por parte del agente educativo. En este es recomendable la flexibilidad, ya que esta característica inclusive da cuenta de su salud mental.

\section{Referencias}

Arévalo, O. M. (1996). Crouis para algún día (Jóvenes de América Latina en los noventa). Pasos, 6 (Número especial), 48-68. Recuperado de http://deicr.org//MG/pdf/6-especial.pdf

Barudy J. (1998). El dolor invisible de la infancia. Una lectura ecosistémica del maltrato infantil. Buenos Aires: Paidós.

Bauman, Z. (2004). Modernidad líquida (3a reimp.). Buenos Aires: Fondo de cultura económica. Recuperado de https://catedraepistemologia.files.wordpress.com/2009/05/modernidadliquida.pdf

Bustelo, E. S. (2007). El recreo de la infancia. Argumentos para otro comienzo. Buenos Aires: Siglo XXI Editores.

De Saint-Exupéry, A. (2009). El principito. Colombia: Editorial Edigrama.

Foucault, M. (1977). Historia de la sexualidad: La voluntad de saber. Madrid: Siglo XXI Editores. Recuperado de http://www.uruguaypiensa.org.uy/imgnoticias/681.pdf

Foucault, M. (1979). Microfísica del poder (2a ed.). Madrid: Las Ediciones de La Piqueta. 
doi: http://dx.doi.org/10.15359/ree.22-2.20

URL: http://www.una.ac.cr/educare

CORREO: educare@una.cr

Hessel, S. (2010). Indígnate (Trad. Colectivo Sáquida). Recuperado de http://www.eldamoneo. com/indignate.pdf

Ovejero A. (2007). Las relaciones humanas. Psicología social teórica y aplicada. Madrid: Biblioteca Nueva. Recuperado de https://telemedicinadetampico.files.wordpress. com/2012/07/83834274-las-relaciones-humanas.pdf

Pinheiro, P. S. (Investigador). (2006). Informe mundial sobre la violencia contra los niños y niñas. Ginebra: Unicef. Recuperado de https://www.unicef.org/lac/Informe Mundial Sobre Violencia 1(1).pdf

Posada-Díaz, Á., Gómez-Ramírez, J. F. y Ramírez-Gómez, H. (2014). Crianza humanizada (2ª ed.). Medellín: Imprenta Universidad de Antioquía.

Unicef. (2006). Convención sobre los derechos del niño. Recuperado de http://www.un.org/es/ events/childrenday/pdf/derechos.pdf

Zuluaga, A. (2015-2016). Proyecto crecer con dignidad. Medellín: Universidad de Antioquia, Centro de Diagnóstico y Derivación. 